\title{
Enabling Synchronous Directional Channel Access on SDRs for Spectrum Sharing Applications
}

\author{
Danh H. Nguyen ${ }^{\dagger^{*}} \quad$ Anton Paatelma ${ }^{\ddagger^{*}} \quad$ Harri Saarnisaari ${ }^{\ddagger}$ \\ Nagarajan Kandasamy ${ }^{\dagger} \quad$ Kapil R. Dandekar ${ }^{\dagger}$ \\ †Drexel University, Philadelphia PA, USA \\ ${ }^{\ddagger}$ Center for Wireless Communications, University of Oulu, Finland \\ \{dnguyen, kandasamy, dandekar\}@drexel.edu \\ \{anton.paatelma, harri.saarnisaari\}@ee.oulu.fi \\ ${ }^{*}$ Co-primary Authors
}

\begin{abstract}
Ubiquitous wireless small-cell deployment requires a fundamental rethink of interference management within the cell, between cells, and with overlaying macrocells. One mean to increase spectral efficiency in these scenarios is through simultaneous directional transmissions and receptions, wherein the antenna directions can be selected such that the overall interference is minimized, or some other cost function is satisfied. To realistically evaluate the performance of these beamsteering techniques, network simulators or testbeds are often required. Nevertheless, a capable testbed that covers sufficient small-cell operational aspects and incorporates directional antennas has yet to be found in the literature. In this paper we present WARP-TDMAC, a software-defined radio framework to enable the prototyping of directionality-based spectrum sharing schemes for small cells. WARP-TDMAC integrates compact pattern-reconfigurable antennas with a high performance 802.11 physical layer and uses a time division multiple access (TDMA) based medium access control (MAC) scheme for antenna direction scheduling. We characterize the synchronization and temporal/spatial scheduling capabilities of this testbed through several example MAC schemes that would have been difficult to realize without our cross-layer framework. The empirical results show that appropriate use of directionality can result in higher network sum rates in dense small-cell deployments, but further investigation is required to find an effective solution for this highly complex operational environment.
\end{abstract}

\section{CCS Concepts}

- Networks $\rightarrow$ Cross-layer protocols; Network experimentation; Mobile networks;

\section{Keywords}

Software-defined radios; small cells; smart antennas; spectrum sharing

Permission to make digital or hard copies of all or part of this work for personal or classroom use is granted without fee provided that copies are not made or distributed for profit or commercial advantage and that copies bear this notice and the full citation on the first page. Copyrights for components of this work owned by others than the author(s) must be honored. Abstracting with credit is permitted. To copy otherwise, or republish, to post on servers or to redistribute to lists, requires prior specific permission and/or a fee. Request permissions from permissions@ acm.org.

WiNTECH'16, October 03-07 2016, New York City, NY, USA

(C) 2016 Copyright held by the owner/author(s). Publication rights licensed to ACM. ISBN 978-1-4503-4252-0/16/10 . .\$15.00

DOI: http://dx.doi.org/10.1145/2980159.2980166

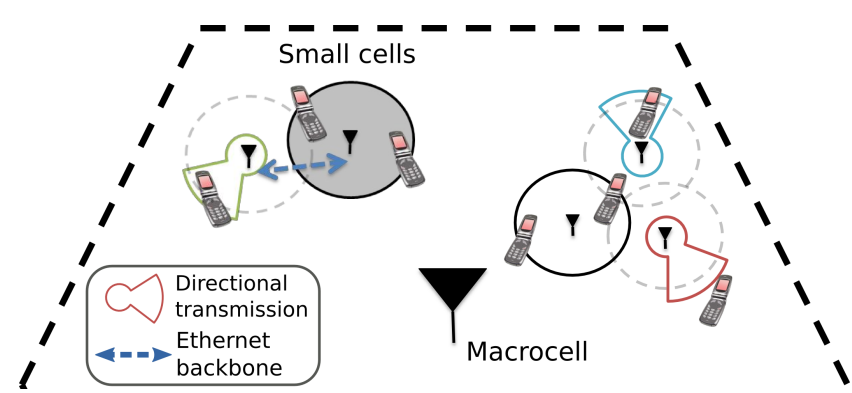

Figure 1: System model of a cognitive small-cell network with high spatial reuse through directionality

\section{INTRODUCTION}

To satisfy the future data demand of mobile users, more efficient methods to utilize the radio spectrum are needed. Network densification, mainly through heterogeneous and small cell (femtocell) deployment, and spectrum sharing have emerged as efficient approaches to enhance spectrum utilization [2]. The radio spectrum can be reused or shared in time, frequency, and space. A licensed band can also become underutilized if it appears vacant in certain time slots or at specific locations. Even in highly utilized spectrum bands, such as those belonging to cellular operators, it remains possible to reuse the spectrum in the spatial domain and improve system throughput. Efficient spectrum sharing presents new opportunities to address the data deluge, while simultaneously posing new challenges in terms of interference management.

In this work we are interested in the possibilities for higher degrees of interference avoidance and spatial packing through directionality. Our focus is on in-band spectrum sharing at different hierarchical levels of a wireless small-cell network to fully utilize the spectrum. At the system level, the spectrum can be shared among small-cell base stations (BSs) belonging to multiple operators. The BSs can operate as equal peers in negotiating spectrum occupancy as well as transmit directions, and possibly coordinate with each other over an Ethernet backbone. Alternatively, the base stations can assume a primary-secondary model wherein the secondary links make use of cognitive radio technologies to opportunistically access the spectrum, provided that a certain signal-to-interference ratio (SIR) is maintained for the primary user. To efficiently utilize the spectrum, we anticipate that coarse timing (packet-level) synchronization 
can be achieved among all base stations and clients through some sync mechanism. As such, our system model mimics a TDMA system, with the distinction that each time slot can be spatially packed with multiple links through directionality. Figure 1 illustrates our system model and eventual deployment goals.

Recent advances in antenna technologies, specifically reconfigurable antennas [10], provide the opportunity to control transmission and interference even after the signals have been up-converted. These antennas can adapt their radiation patterns in response to the changing environment and thus offer an additional degree of freedom to enhance small-cell networks for a higher degree of spatial aggregation. However, these new technologies have not been integrated into existing wireless testbeds for an experimental study in the context of small cells.

Our contributions in this paper are twofold: First, we develop a software-defined radio (SDR) small-cell testbed on top of the Wireless open-Access Research Platform (WARP) [1]. This testbed, called WARP-TDMAC, integrates compact, electronically reconfigurable antennas into WARP physical layer and implements a custom TDMA-style MAC layer to provide timing, synchronization, resource scheduling, and programmable directionality to all radio nodes. To the best of our knowledge, this is the first experimental testbed to offer real-time scheduling in both time slotted channel access and antenna beam direction at millisecond granularity. Second, using this testbed, we experimentally demonstrate the feasibility of dense spatial packing of multiple synchronous small-cell links through directional transmission and reception. Our measurement results through an exhaustive directionality sweep in a two-link spectrum sharing scenario show that proper use of directionality can increase network sum rates by $68 \%$. This potential motivates further development of low-overhead signaling schemes to realize the performance gains promised by opportunistic spectrum sharing.

The paper is organized as follows. Sec. 2 surveys related work in cognitive radio testbed development. Sec. 3 describes WARP-TDMAC's system architecture. Sec. 4 presents our experimental results, and we conclude the paper in Sec. 5 .

\section{RELATED WORK}

Related work to ours falls in the two areas below.

Achieving Deterministic Timing on SDRs: It is well known that due to the physical layer programmability of software-defined radios, achieving deterministic packet timing and processing latencies proves to be difficult [12]. To address the real-time processing demands of modern wireless standards, recent SDR architectures emerge with highly optimized processing pipelines and span three subgroups: software-based, hardware-based, and hybrid approaches. Software-based solutions, such as SORA [15] and Ziria [13], implement the PHY implementation purely in software running on a CPU and employ various optimization techniques to meet protocol timing constraints. The proprietary nature of these optimizations makes it difficult to extend such platforms and build protocol variants. Alternatively, recent work $[3,5]$ attempts to move a larger portion of PHY and MAC processing onto hardware and proposes a split-functionality hybrid architecture. The key idea is to carry out time-critical operations, such as carrier sensing and backoff timers, on the FPGA hardware for precise timings, while letting the rest of signal processing proceed in software in a non-deterministic manner. This approach requires careful orchestration of multiple processing elements to prevent data loss and maintain inter-layer consistency.

For a synchronous directional small-cell testbed, we put emphasis on the radio node's ability to operate in real time with deterministic timelines and opted for hardware-based SDR architectures. These solutions implement the entire physical layer on programmable hardware logic, such as FPGAs, while making their implementations amendable to changes by end users. The WARP platform [1], with its 802.11 Reference Design, is a prominent example of this approach. While WARP's hardware design provides the base for our small-cell testbed, we modify it extensively to support scheduled channel access with directionality. We also note that the few existing TDMA implementation efforts on WARP that we can find either $i$ ) operate in non-real time with the requirement of an Ethernet backbone for synchronizing transmissions [6], or ii) do not disclose the sync mechanism, achieved precision, or any scheduling capability [16]. This work builds on these efforts to realize over-the-air (OTA) synchronization and fine-grained temporal/spatial scheduling without the need for a wired backbone.

Integrating Beam-Steerable Directional Antennas into Wireless Testbeds: Compared to omni-directional antennas, directional antennas bring about many advantages, such as higher transmission gains, lower interfererence, and more opportunities for spatial reuse. Numerous directional MAC protocols have been proposed in the literature (see [4] for a survey), but only a few are evaluated on actual testbeds. Notable prototype-driven studies include DIRC [7], Mobisteer [9], and $2 \mathrm{P}$ [11]. These testbeds leverage commercial off-the-shelf Atheros WiFi chipsets with modified 802.11 MAC firmware (often based on the open-source MadWiFi driver) to perform directional variants of carrier-sense channel access. The DIRC prototype [7] suppresses carrier sensing and link-layer ACKs to enable TDMA-based scheduling over an Ethernet backbone. However, due to the lack of PHY layer access, it can only achieve limited time slot resolution of $4 \mathrm{~ms}$, and directional reconfiguration happens at an even slower time scale, on the order of tens of milliseconds. More recently, research interests in millimeter-wave (mmWave) wireless technologies have also prompted the development of SDR testbeds with mmWave frontends and highly directional antennas, such as WiMi [14]. These testbeds are mainly equipped with mechanically steerable horn antennas and are geared more toward channel sounding experiments in an offline flow. In contrast to the above prototypes, WARPTDMAC operates in real time, has tight intergration with compact, electronically reconfigurable antennas and a modifiable physical layer, as well as integrates scheduling in both time and antenna directions at the millisecond level.

\section{SYSTEM ARCHITECTURE}

The goal of WARP-TDMAC is to provide a real-time platform for studying the intricate cross-layer and co-layer interference patterns of small-cell networks, as well as investigating possible spatial packing techniques using directionality and efficient resource scheduling. In this section we describe the architectural details of this testbed, including its integrated reconfigurable antennas, FPGA-based 802.11 physical layer, and associated TDMA-based MAC layer. An overview of our hardware testbed is shown in Fig. 2. 


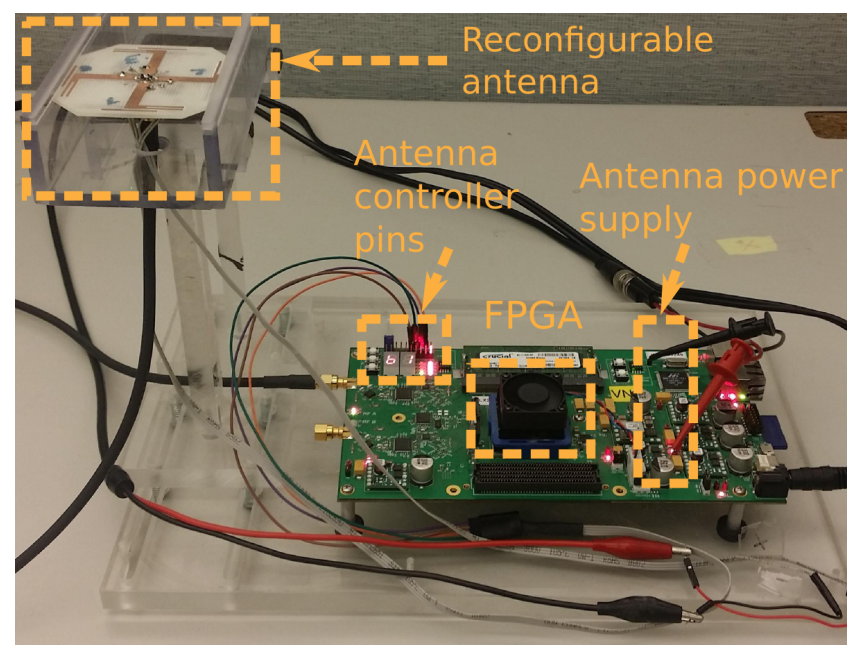

Figure 2: WARP-TDMAC testbed hardware

\subsection{PHY Layer and Reconfigurable Antennas}

Our interest is on femtocell networks employing orthogonal frequency-division multiple access (OFDMA) physical layer, primarily due to its multi-user interference avoidance properties and robustness to multipath. In OFDMA, the available spectrum band is divided into orthogonal groups of subcarriers, commonly referred to as subchannels. OFDMA works as a multiple access technique by assigning specific patterns of subchannels in the time-frequency space to different users [8]. Therefore, OFDMA femtocells can exploit channel variations in both frequency and time domains for interference avoidance.

As a starting point for development, WARP-TDMAC makes use of the open-source physical layer from the 802.11 Reference Design [1]. This design implements in FPGA fabric a real-time, single-input single-output (SISO), 802.11a/g standard compliant PHY transceiver. While this PHY can achieve interoperability with commercial WiFi devices, it lacks the ability to carry out OFDMA multi-user access functionalities. Our testbed emulates the functionality by implementing a TDMA/OFDM system with multi-user scheduled access in time. Furthermore, in addition to the traditional time-frequency resource allocation, we specifically enable spatial domain scheduling by incorporating reconfigurable antenna controllers and precise hardware timers into the PHY.

In this work we achieve dynamic directionality through compact pattern-reconfigurable antennas. These antennas provide inherent space and cost benefits by integrating many switchable radiating elements on a single device, thereby enabling steerable directed beams with a smaller number of RF chains and lower processing overhead than phased array and adaptive systems. These properties make reconfigurable antennas an ideal technology to be integrated into mobile devices and SDR testbeds. Leveraging our previous work with reconfigurable Alford-loop antenna (RALA) design [10], we integrate this antenna into WARP-TDMAC. The antenna's internal structure and available beam patterns are depicted in Fig. 3. Our RALA can radiate in both an omnidirectional pattern as well as four directed beams at $90^{\circ}$ separation with controllable beam widths. This unique omni/directional reconfigurability of RALAs provides the
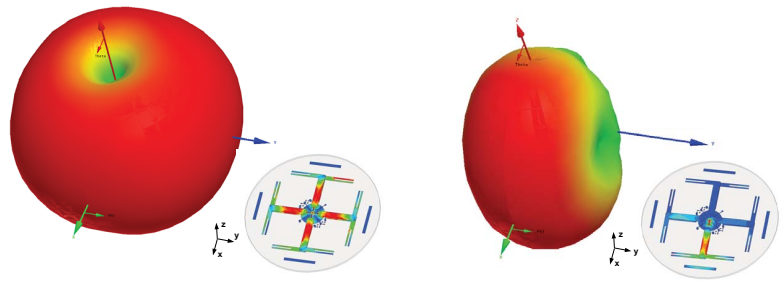

(a)
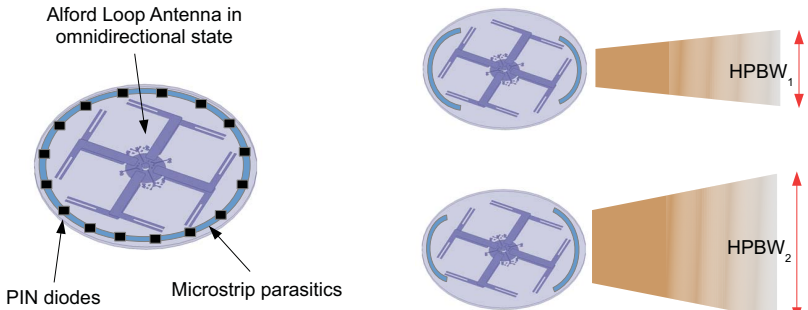

(b)

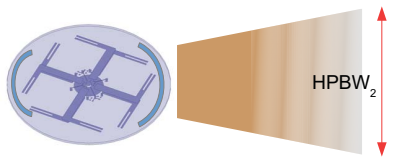

Figure 3: (a) RALA with omni (left) and directional (right) patterns. (b) Antenna design and beam characteristics

fundamental building block for spatial scheduling operations: synchronization and control signals are broadcasted using the omnidirectional antenna mode, while unicast transmissions can be spatially packed using directional antenna configurations. RALA's detailed architecture, as well as its beam and gain specifications, can be found in [10].

Reconfigurable antennas are integrated into the physical layer through the generic 16-pin general purpose input/output (GPIO) debug header (readily available on WARP hardware) and a Xilinx axi_gpio IP core. This core is controlled by the Microblaze via a shared register and interfaces directly with the antenna control circuitry, which in turn sets the necessary voltages for antenna elements to generate desired patterns. From the point of view of MAC layer software, the latency of antenna pattern switching is mainly induced by the GPIO register write, which is on the order of one AXI bus transaction ( 32 clock cycles, or $\sim 0.2 \mu \mathrm{s}$ ). The antenna controller is kept separately from the Tx and Rx chains so that its control logic can be independent of ongoing transmissions. Antenna reconfiguration can happen in every time slot and on a per-packet basis, immediately prior to any scheduled transmission or reception in that slot.

\subsection{MAC Layer}

WARP-TDMAC's software framework includes the antenna controller and PHY hardware abstraction layers (HALs), a customizable TDMA-based MAC layer, as well as upperlayer functionalities. We implement these components on a dual-core architecture with two embedded Microblaze soft processor cores. One processor is responsible for time-critical MAC functionalities, such as synchronous channel access and resource schedule processing. The other core is responsible for inter-packet behaviors that are not time sensitive, including Ethernet communications with other nodes, local traffic generation, and logging functionalities. A high level overview of the software architecture is depicted in Fig. 4.

The MAC layer implements necessary mechanisms for physical layer control, time synchronization, time-frequency-space resource block scheduling, packet buffering, and external sup- 


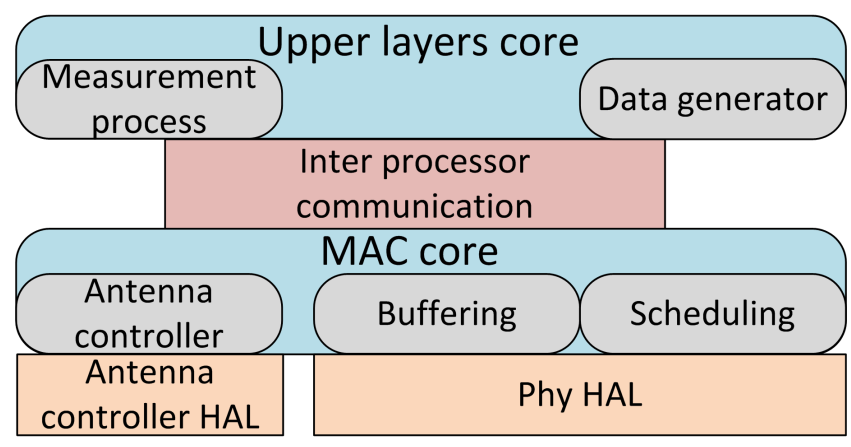

Figure 4: WARP-TDMAC system architecture

port for application-specific MAC code. Outside of these core functionalities, the actual MAC frame structure, antenna control logic, and resource scheduling algorithm can all be modified in the application-specific MAC code. This modularity makes it straightforward for users to experiment with different small-cell specific MAC functionalities. Our current MAC implementation employs a centralized synchronization source coupled with independent network-specific scheduling. A single main base station (BS) (e.g., a macrocell or primaryuser femtocell) provides the reference synchronization signal for all radios to obtain a common time base. On the other hand, resource allocation is handled independently by each network operator through its own small-cell BSs. Within a network, scheduling decisions can be centralized at a specific base station, or distributed among network nodes to enable ad hoc, device-to-device communications.

The MAC layer software, implemented in $\mathrm{C}$ and running on the "lower" Microblaze core, consists of two parts: interruptdriven and main loop code. Interrupt-based code implements time-critical routines that handle network time synchronization, resource scheduling, antenna control, and other timesensitive processing tasks. The main loop code contains PHY-layer callbacks for packet transmission and reception, as well as routines for inter-processor communications, MAC packet buffering, and processing of user inputs. The key subsystems of our MAC layer are described below.

\subsubsection{Time Synchronization and Network Roles}

In WARP-TDMAC, coarse level network-wide synchronization is carried out through a Sync packet sent by the main base station at the beginning of each TDMA frame. This Sync control packet signifies all nodes in the vicinity to simultaneously start their hardware timers to achieve a common time base. The lower MAC code constantly polls the Rx packet queue to detect a Sync reception and read the PHY-supplied microsecond timestamp indicating the start of this reception (defined in the 802.11 standards as the instant when the SIGNAL field is successfully decoded). Once the Sync packet reception timestamp is obtained, we consider the node synchronized with the main base station and configure its hardware timer to start the periodic interrupt at the beginning of each time slot. Assuming negligible propagation delay, as is typical in an indoor environment where femtocells operate, this technique can achieve microsecond-level synchronization with high fidelity among all network nodes.

Another challenge is for the main base station to also synchronize with the rest of the network despite the lack of a Sync reception timestamp (since it is busy sending the
Sync signal). We overcome this by empirically measuring the processing delay (through hardware debug pins and an oscilloscope) between the time the main BS's MAC code hands over the Sync packet for PHY transmission and the time packet reception starts at a receiving node. This period, measured to be $27 \mu \mathrm{s}$, usually remains constant and therefore does not require recalibration. We add this additional postSync delay only at the main base station in the hardware timer's wait time to interrupt at the next slot boundary.

Each WARP-TDMAC node has three modes of operation: main BS, slave BS, or client, decided at boot time by user inputs via the DIP switches. As mentioned previously, there is only a single main base station, which emulates the role of a macrocell or primary-user femtocell and provides the central timing source. There can be an arbitrary number of slave (small-cell) BSs and clients, potentially belonging to multiple operator networks. The main base station always operates in Sync mode, i.e., it constantly assumes synchronization with the rest of the radio nodes. Slave BSs and clients can operate in both Sync and Non-Sync modes. They are automatically set to Sync mode after receiving a Sync signal and to NonSync mode if they fail to receive a new Sync signal following the end of a TDMA frame.

\subsubsection{Scheduling Framework}

A WARP-TDMAC frame starts with a Sync signal from the main base station, followed by a number of Physical Resource Blocks (PRBs), and ends with a Guard time slot. Each PRB is further broken down into two time slots which can be used to transmit different types of packets. We use this dual time slot aggregation in forming PRBs to decrease the granularity of resource scheduling and incurred protocol overhead. Our current implementation uses 1.6ms MAC time slots to accommodate long packet transmissions.

Based loosely on existing LTE standards, WARP-TDMAC supports four resource block types: Beacon, Ack, Data, and Empty. Beacon resource blocks are reserved for base stations to send BEACON control packets which carry resource schedules, link statistics, and other control information. Ack resource blocks are used by clients to send ACK packets for acknowledging data reception, as well as establishing presence to the BSs. ACK packets can also hold additional information about the clients' link states. Note that each control packet occupies only a portion of a single time slot, freeing up the other time slot in the PRB for additional data transmission. Data resource blocks are used purely for data transfer in either up- or downlink directions. An Empty resource block denotes an unallocated block, often used to suppress communications and yield a PRB to other links.

An example WARP-TDMAC frame is shown in Fig. 5. Each base station broadcasts its resource schedule through its own Beacon packet, which may be overheard by other BSs as well. For efficiency, we orthogonalize in time all control packets (Beacons and Acks) by network or link group within the TDMA control time slots. For example, beacons from base stations of different networks (or link groups) occupy the same Beacon time slot, but they further partition the slot into smaller slices to transmit orthogonally. Only data packets are allowed to be scheduled in overlapping time periods. This allows us to evaluate different spatial reuse strategies in Data slots, while reserving an interference-free control channel for protocol coordination and link state exchanges. 


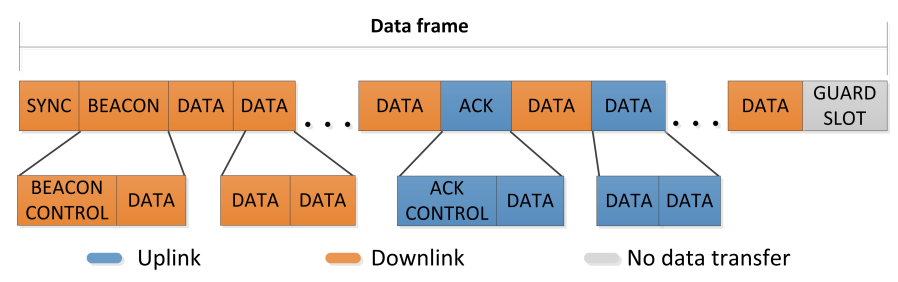

Figure 5: Example WARP-TDMAC frame structure

\subsubsection{MAC Packet Buffers}

WARP-TDMAC needs a packet buffer system at the MAC layer to support opportunistic spectrum sharing operations. Resource scheduling decisions are often made at the beginning of a TDMA frame, so having a sufficient MAC packet buffer will enable a base station to make informed scheduling decisions based on its expected traffic load and enable support for quality of service (QoS).

Our MAC layer uses a dual-buffer system, one each for the Tx and Rx paths. These buffers are implemented in hardware using a $128 \mathrm{~KB}$ dual-port RAM and constitute a shared memory region that is mapped into the address spaces of both processor cores. On the Rx path, a received data packet is moved immediately from the PHY $\mathrm{Rx}$ buffer to MAC $\mathrm{Rx}$ buffer following a successful reception, and then made available to upper layers. On the Tx path, we implement a buffer management system to manage many packets at once via a series of transmit queues. Each queue is a doubly linked list data structure that maintains a specific logical order of the buffered Tx packets. Packets can be dequeued and sent to PHY Tx buffer in any manner as specified by the QoS scheduling algorithm. Cross-layer packet transfers between MAC and PHY buffers are handled using Direct Memory Access (DMA). This buffering system enables great flexibility in WARP-TDMAC resource scheduling, as well as QoS support alongside with maximizing spectral efficiency.

\subsubsection{PHY Access and Radio / Antenna Controls}

Many resource scheduling algorithms can greatly benefit from radio-level information. Examples include user scheduling to leverage channel diversity, received signal strength indication (RSSI) based cell handoffs, and opportunistic spatial reuse. Our 802.11-based PHY provides a lot of useful metadata for each received packet, including reception timestamp, channel coefficient estimates, RSSI, and automatic gain control (AGC) settings. WARP-TDMAC provides easy access to this information via a set of APIs. In addition, the MAC packet buffer system propagates this radio metadata as it shuttles Rx packets from PHY, making it available to upper layers as well.

Cognitive resource allocation schemes often require cooperation from the physical layer for actions such as subchannel access, antenna reconfiguration, and power control. To efficiently carry out these decisions within a small time window, the MAC layer needs fine-grained control of radio resources on a per-packet basis. We enable this by implementing a set of radio and antenna controller APIs, as well as leveraging the metadata fields of each PHY Tx buffer slot to pass desired operating parameters for the PHY to carry out during transmission. These mechanisms enable the MAC to control physical layer resources at various levels of granularity, either on a WARP-TDMAC frame or per-packet basis.

\subsection{Upper Network Layers}

In WARP-TDMAC, a separate Microblaze processor core executes all upper layers' functionalities, including traffic generation, logging, and measurement routines. The upper layer software is rather simple and mostly reserved for application-specific operations. By default it only includes a local traffic generator, inter-processor communications (IPC) routines, and a system tick timer running at $1 \mathrm{kHz}$. To support application needs, additional hardware resources and drivers are included for use by the upper layer software, including an external SO-DIMM DDR3 RAM memory controller, Ethernet peripherals for backbone network support, SD card file system access for log and measurement data storage, and an interrupt controller for DMA operations.

\section{EXPERIMENTAL EVALUATIONS}

This section presents our verification and spatial packing experiments with WARP-TDMAC.

\subsection{Synchronization and Scheduling}

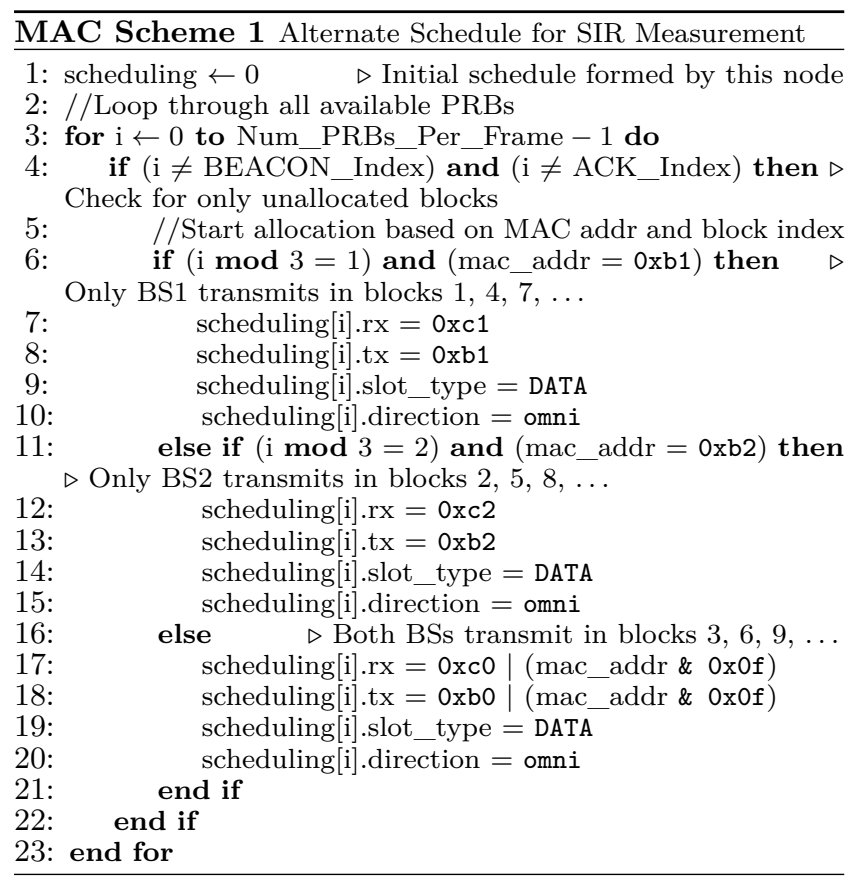

To verify our testbed's synchronization and scheduling capabilities, we set up an alternating link schedule MAC scheme typically used to measure signal-to-interference ratios (SIRs) during concurrent in-band transmissions. This channel access scheme, depicted as MAC Scheme 1, alternates PRB allocations among three access scenarios: exclusive primary-user downlink, exclusive secondary-user downlink, and interfering downlink transmissions. Each client receiver can measure the received powers (e.g., through RSSIs and AGC settled gains) from both its home BS and interfering BS during the first two scenarios, and estimate its SIR. The estimated SIR can then be used to cross-reference and validate results in the interfering transmission scenario. With the short resource block time supported by WARP-TDMAC (1 PRB equals 2 time slots, or $3.2 \mathrm{~ms}$ ), all three access scenarios can be carried out back to back within $10 \mathrm{~ms}$, reasonably below the WiFi channel coherence time. In Sec. 4.2, we use the 


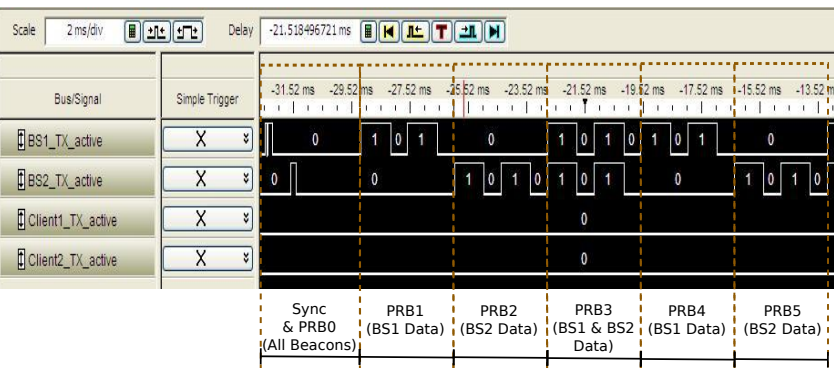

(a) 20ms logic analyzer capture of TX PHY Active debug signals from all WARP nodes in a WARP-TDMAC frame scheduled according to MAC scheme 1

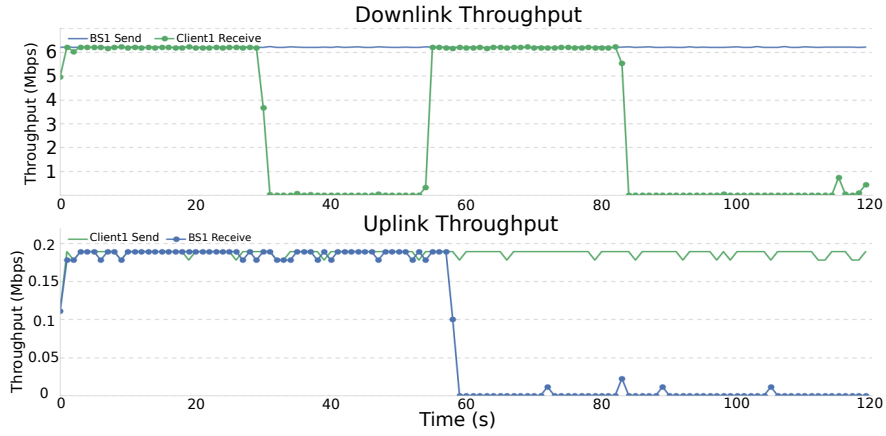

(b) Primary link throughput as a time series. 0-30s: no second-user IF; 30-55s: downlink-only IF; 56-84s: uplink-only IF; 84-120s: IF in both directions. All nodes use omnidirectional antennas.

Figure 6: Synchronization and scheduling experimental results

same MAC scheme to measure the SIRs of the primary- and secondary-user links in spatial packing scenarios with omni and directional antenna modes.

WARP hardware design routes various internal MAC/PHY signals to the 16-pin debug header to allow monitoring of MAC/PHY state at runtime using an oscilloscope or logic analyzer. Figure 6 a shows a $20 \mathrm{~ms}$ logic analyzer capture of the Tx PHY Active debug signals from all WARP nodes in a WARP-TDMAC frame scheduled using MAC Scheme 1. This signal asserts when the Tx PHY begins transmitting a frame and de-asserts after the last sample is sent. We observe that WARP-TDMAC executes our scheduling logic consistently with tight synchronization.

For real-time OTA system verification, we saturate the channel with primary-user downlink and uplink traffic (in their respective PRBs). Then we assemble four other synchronous access MAC schemes specifically for the secondary BS2-Client 2 link: $i$ ) complete yielding (no transmission), ii) downlink-only interference (BS2 transmits concurrently in all downlink PRBs), iii) uplink-only IF (Client 2 transmits concurrently in all uplink PRBs), and iv) IF in both directions (both transmits in respective PRBs). These MAC schemes are programmed into our SDR framework as applicationspecific $M A C$ code running in the lower MAC core (see Fig. 4), which can be activated or disabled on the fly using push buttons on the WARP boards. Figure $6 \mathrm{~b}$ depicts the primary-user link-layer throughput captured over a window of $120 \mathrm{~s}$, with deliberate interference from the secondaryuser link at times. All nodes use omnidirectional antennas in this experiment. The resulting graphs verify WARPTDMAC's ability to prototype high-speed wireless small-cell links, yielding a nominal rate of $6.2 \mathrm{Mbits} / \mathrm{s}$ downlink and $0.18 \mathrm{Mbits} / \mathrm{s}$ uplink throughput in this allocation scheme. Furthermore, synchronous channel access can also be realized in WARP-TDMAC through scheduling, as seen through the time-division duplexing (TDD) interference patterns, i.e. independent downlink and uplink interference can be generated. The modularity and flexibility of WARP-TDMAC system architecture is also evident, allowing different MAC schemes to be swapped in and out at runtime.

\subsection{Spatial Packing Measurements}

In this section we present our over-the-air measurement results to demonstrate the potential for dense spatial reuse with reconfigurable directional antennas in an interferencelimited environment.

\subsubsection{Experimental Methodology}

We consider a spectrum sharing case of two co-channel small-cell links, operating independently and possibly belonging to different operators (thereby not cooperating). The small-cell links access the channel synchronously and interfere with each other in every Data time slot (control packet transmissions are orthogonal in their own PRB). The nodes are scattered in an open atrium with a distance of $10 \mathrm{~m}$ between the two BSs. Each BS is serving its intended client (labeled as $\mathrm{C} 1$ and $\mathrm{C} 2$ ) that is situated $3 \mathrm{~m}$ away. The measurement topology is depicted in Fig. 7. Reconfigurable antennas are equipped at all network nodes, enabling them to switch among the omnidirectional mode and four available directional modes at any time. Our measurement procedure performs autonomous and distributed directionality sweeps: based on the frame_number field carried in the Sync signal at the start of each TDMA frame, all nodes switch to the appropriate antenna configuration used for that entire frame. We iteratively sweep through all antenna configurations of interest, including the all-omnidirectional mode (i.e., all nodes use the omnidirectional pattern) and 256 directional modes (4 directional modes at each communicating party). For simplicity, we omit the cases with mixed omni and directional antenna modes used during Data PRBs. For each antenna configuration, we collect the packet delivery ratios (PDRs) and downlink throughputs of both small-cell links. Each TDMA measurement frame contains 34 downlink packets (spreading across all available Data PRBs), and each antenna configuration is active for 20 TDMA frames, yielding a total of 680 downlink packets per antenna configuration. The PHY is fixed in WiFi channel $14(2.484 \mathrm{GHz})$, with $15 \mathrm{~dB}$ Tx RF gain, QPSK modulation, and code rate $1 / 2$, yielding a consistent PHY rate of $12 \mathrm{Mbits} / \mathrm{s}$.

Scheduling antenna directions at finer time scales (i.e., physical resource block or time slot level) is also possible through WARP-TDMAC link scheduling mechanism; see the pseudocode for SIR measurement in MAC Scheme 1 as an example. We collect SIR readings at each receiver (client) using this scheduling scheme for all antenna configurations in the sweep to cross-reference with the spatial packing throughput results. Nevertheless, doing so requires each 


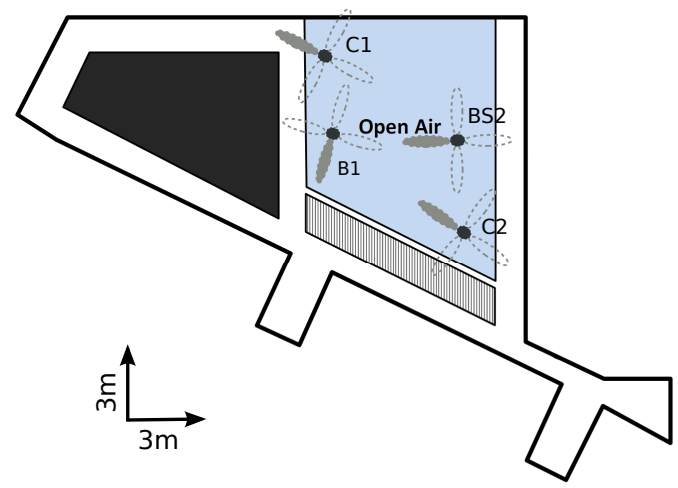

Figure 7: Measurement setup for the spatial reuse experiment

small-cell base station to assemble and broadcast its own directional link schedule for each TDMA frame.

To assess the spatial packing potential, we compare allomnidirectional transmission to the best and worst directional transmission scenarios. For fair comparison, we ensure that the total power radiated out of each transmit antenna is equivalent for all schemes. Using a vector network analyzer, we measure the return losses of the different operating modes of the reconfigurable Alford-loop antennas. The omnidirectional mode has a return loss of $-9 \mathrm{~dB}(\sim 12.5 \%$ of incident power), while the four directional modes have much higher return losses of $-3 \mathrm{~dB}$ ( $50 \%$ of incident power). Thus, we normalize the directionally radiated power to that of the omnidirectional case by increasing the incident power hitting the antenna in directional modes by $75 \%$. This is accomplished by compensating all directional antenna modes with an additional $2.5 \mathrm{~dB}$ Tx RF gain.

\subsubsection{Experimental Results}

Figure 8 shows the mean SIRs and standard deviations at the two clients, as well as the average per-link SIR in different antenna configurations. The best and worst directional modes here are determined based on the average per-link SIR at both clients. The best directional mode has a $6 \mathrm{~dB}$ loss in per-link SIR compared to the all-omnidirectional case. In addition, the primary BS1-C1 link also experiences a 13 $\mathrm{dB}$ reduction in SIR. However, the whole network achieves a more balanced interference condition with the secondary link gaining its SIR by $8 \mathrm{~dB}$. In contrast, the worst directional mode greatly degrades the SIRs at both clients.

The MAC-layer throughputs of both small cell links, collected for 20 TDMA frames (one measurement round), are depicted in Fig. 9. Since the measurement procedure in the upper layer generates packets for transmission with a fixed-size MSDU of 1460B, the PDRs are directly proportional to MAC layer throughputs and thus not shown. In the all-omnidirectional case, only the primary link (BS1-C1) can achieve a non-zero PDR and consequently a downlink throughput of $4.92 \mathrm{Mbps}$ ( $77 \%$ of the link maximum). The secondary link cannot successfully deliver any packet and has no useful throughput. The best directional mode improves the throughputs of both links significantly despite their strong mutual interference. Specifically, the primary link can increase its PDR from 0.77 to 1.00 , while the secondary link can also deliver a useful PDR of 0.30. As a result, per-link throughput (and therefore network sum rate)

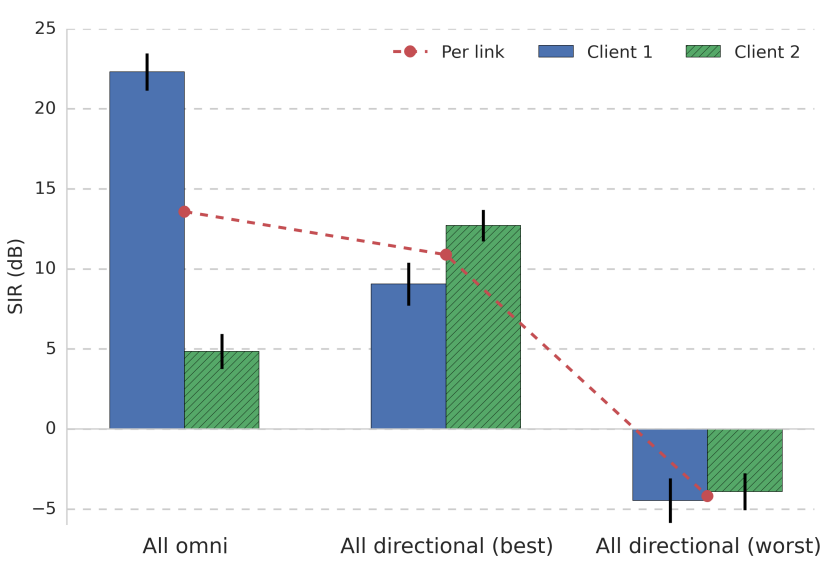

Figure 8: SIR at each client in the two-link interference scenario

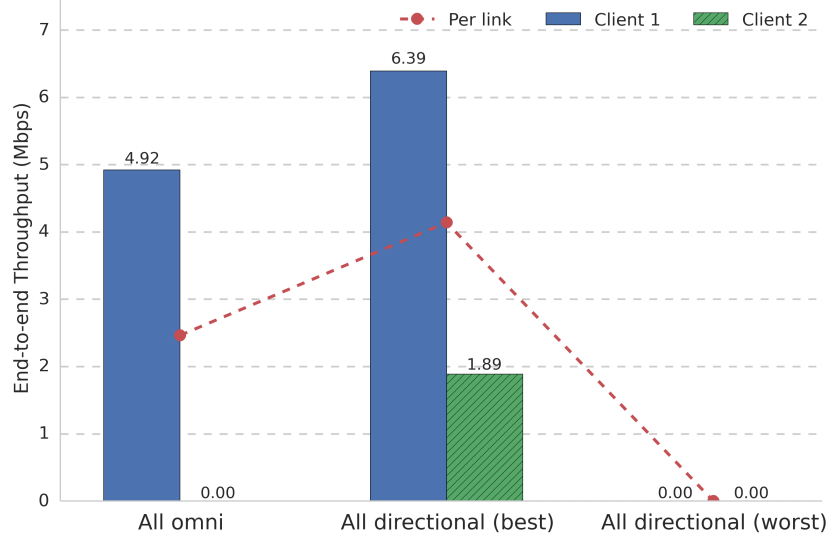

Figure 9: Link throughput at each client in the twolink interference scenario

increases by $68 \%$ compared to the all-omnidirectional mode, from 2.46 Mbits/s to $4.14 \mathrm{Mbits} / \mathrm{s}$. Though a suboptimal directional configuration can result in zero throughputs on both links in the worst case, a robust antenna selection algorithm can help avoid this undesired scenario and achieve the spatial reuse gains above.

\section{CONCLUSION}

In this paper we have described WARP-TDMAC, a crosslayer SDR framework to enable prototyping of cognitive small-cell networks with synchronous and directional channel access. WARP-TDMAC integrates compact, electronically reconfigurable antennas with a high performance physical layer and provides mechanisms for efficient resource scheduling in both temporal and spatial domains at millisecond slot time granularity. Using WARP-TDMAC, we experimentally demonstrated the values of antenna directionality in typical small-cell spectrum sharing scenarios. Our results show significant increases in network sum rates when using directionality as an interference management technique. In future work, we plan to investigate efficient methods for autonomous selection of optimal antenna states, as well as low-overhead protocols for spatial packing of links through directionality. 


\section{ACKNOWLEDGMENTS}

We thank Yuqiao Liu for his help in assembling the reconfigurable antennas. This work was performed as part of the WiFiUS (Wireless Innovation between Finland and US) program and was supported by the National Science Foundation under grant 1457306 as well as Tekes under Grant Dnro $2336 / 31 / 2014$.

\section{REFERENCES}

[1] WARP Project. http://warpproject.org.

[2] J. G. Andrews, S. Buzzi, W. Choi, S. V. Hanly, A. Lozano, A. C. K. Soong, and J. C. Zhang. What will $5 \mathrm{G}$ be? IEEE Journal on Selected Areas in Communications, 32(6):1065-1082, 2014.

[3] B. Bloessl, A. Puschmann, C. Sommer, and F. Dressler. Timings Matter: Standard Compliant IEEE 802.11 Channel Access for a Fully Software-based SDR Architecture. In Proceedings of ACM WiNTECH'14, pages 57-64, 2014.

[4] H.-N. Dai, K.-W. Ng, M. Li, and M.-Y. Wu. An Overview of Using Directional Anatennas in Wireless Networks. International Journal of Communication Systems, 26(4):412-448, 2013.

[5] P. Di Francesco, S. McGettrick, U. K. Anyanwu, J. C. O'Sullivan, A. B. MacKenzie, and L. a. DaSilva. A Split MAC Approach for SDR Platforms. IEEE Transactions on Computers, (4):1-1, 2014.

[6] S. R. Kandukuri. Power Control Mechanisms on WARP Boards. Technical report, Mid Sweden University, 2013.

[7] X. Liu, A. Sheth, M. Kaminsky, K. Papagiannaki, S. Seshan, and P. Steenkiste. DIRC: Increasing Indoor Wireless Capacity using Directional Antennas. In Proc. of ACM SIGCOMM '09, 2009.

[8] D. Lopez-Perez, A. Valcarce, G. De La Roche, and J. Zhang. OFDMA femtocells: A roadmap on interference avoidance. IEEE Communications Magazine, 47(9):41-48, 2009.

[9] V. Navda and A. P. Subramanian. MobiSteer: using steerable beam directional antenna for vehicular network access. In Proc. of ACM MobiSys '07, 2007.

[10] D. Patron and K. R. Dandekar. Planar reconfigurable antenna with integrated switching control circuitry. In Proc. of the 8th European Conference on Antennas and Propagation (EuCAP 2014), pages 2737-2740, 2014.

[11] B. Raman and K. Chebrolu. Design and Evaluation of a new MAC Protocol for Long-Distance 802.11 Mesh Networks. In Proc. of ACM Mobicom '05, pages 156-169, 2005.

[12] T. Schmid, O. Sekkat, and M. Srivastava. An experimental study of network performance impact of increased latency in software defined radios. In Proc. of ACM WinTECH 'O7, pages 59-66, 2007.

[13] G. Stewart, M. Gowda, G. Mainland, B. Radunovic, D. Vytiniotis, and C. L. Agullo. Ziria: A DSL for Wireless Systems Programming. In Proc. of ASPLOS '15, pages 415-428, 2015.

[14] S. Sur, V. Venkateswaran, X. Zhang, and P. Ramanathan. $60 \mathrm{GHz}$ Indoor Networking through Flexible Beams : A Link-Level Profiling. In Proc. of ACM SIGMETRICS 15, pages 71-84, 2015.

[15] K. Tan, J. Zhang, J. Fang, H. Liu, Y. Ye, S. Wang, Y. Zhang, H. Wu, W. Wang, and G. M. Voelker. Sora : High Performance Software Radio Using General Purpose Multi-core Processors. In Proc. of USENIX NSDI'09, 2009.

[16] H. Tuomivaara and M. Raustia. Demonstration of Distributed TDMA MAC Protocol Implementation with OLSR on Linux Enriched WARP. In Proc. of ACM Mobicom '09, pages 85-86, 2009. 